\title{
Correction to: Psychophysical detection and learning in freely behaving rats: a probabilistic dynamical model for operant conditioning
}

\author{
İsmail Devecioğlu ${ }^{1}$ (D) $\cdot$ Burak Güçlü ${ }^{2}$ \\ Published online: 23 July 2020 \\ (C) Springer Science+Business Media, LLC, part of Springer Nature 2020
}

\section{Correction to: Journal of Computational Neuroscience} https://doi.org/10.1007/s10827-020-00751-8

The original version of this article unfortunately has some typographical errors in equations (5), (6), (7), (8), and (12). Apparently, the corrections during the proofing stage were not accurately implemented; some equations were further corrupted after editing.

Thus, this erratum is presented to fix these errors, providing the correct equations.

- Equation (5):

$$
W_{z} \sim N\left(\overline{w_{z}}, c \overline{w_{z}}\right), \quad B_{z} \sim N\left(\overline{b_{z}}, c \overline{b_{z}}\right)
$$

- Equation (6):

$$
c= \begin{cases}0.5, & \text { for 6-parameter model } \\ \text { constant to be fitted, } & \text { for 7-parameter model } \\ b b(t / 10)^{b p}, & \text { for 8-parameter model }\end{cases}
$$

The online version of the original article can be found at https://oi.org/ 10.1007/s10827-020-00751-8

Burak Güçlü

burak.guclu@boun.edu.tr

1 Biomedical Engineering Department, Tekirdağ Namık Kemal University, 59030 Tekirdağ, Turkey

2 Institute of Biomedical Engineering, Boğaziçi University, Kandilli Campus, Çengelköy, 34684 Istanbul, Turkey
- Equation (7):

$$
M_{l}=\left\{\begin{array}{cc}
0, & E_{l}<E_{r} \\
1, & E_{l}>E_{r}
\end{array}, \quad M_{r}=\left\{\begin{array}{cc}
1, & E_{l}<E_{r} \\
0, & E_{l}>E_{r}
\end{array}\right.\right.
$$

- Equation (8):

$\rho=\left\{\begin{array}{l}0, \text { miss }\left(S=1 \text { and } M_{l}=1\right) \text { or false alarm }\left(S=0 \text { and } M_{r}=1\right) \\ 1, \text { hit }\left(S=1 \text { and } M_{r}=1\right) \text { or correct rejection }\left(S=0 \text { and } M_{l}=1\right)\end{array}\right.$

- Equation (12):

$X_{S}=\left\{\begin{array}{ll}0, & S=0 \\ 1, & S=1\end{array}, \quad X_{N S}= \begin{cases}1, & S=0 \\ 0, & S=1\end{cases}\right.$

The original article has been corrected.

Publisher's note Springer Nature remains neutral with regard to jurisdictional claims in published maps and institutional affiliations. 